\title{
Robotic Thumb Grasp-Based Range of Motion Optimisation
}

\author{
Konstantinos Dermitzakis, Andreas Ioannides and Hwai-ting Lin
}

\begin{abstract}
With the thumb serving an important role in the function of the human hand, improving robotic prosthetic thumb functionality will have a direct impact on the prosthesis itself. So far, no significant work exists that examines the ranges of motion a prosthetic thumb should exhibit; many myoelectric prostheses arbitrarily select them. We question this design practice as we expect a significant functional volume reduction for performing certain activities vs. the maximum obtainable workspace. To this end, we compare and contrast four anatomically-accurate thumb models. We quantify their angular ranges of motion by generating point clouds of endeffector positions, and by computing their alpha-shape bounded volumes. Examining the function of the thumb for several grasps, we identify a $76 \%$ reduction of the required workspace volume vis-a-vis the maximum volume of a "generic"' human thumb.
\end{abstract}

\section{INTRODUCTION}

The loss of an upper-limb, or part thereof, causes a sudden and dramatic reduction in the ability of the affected person to (1) perform certain Activities of Daily Living (ADLs), (2) sense their surroundings, and (3) causes a major change to their cosmetic appearance [1]. As such, many upperextremity amputees seek to compensate this loss by wearing a prosthesis. However, rejection rates for upper-limb myoelectric prostheses are still high [2] and substantial progress remains to be made in order to achieve an acceptable level of functionality by the over-whelming majority of prosthesis users.

The three most common reasons leading to myoelectric prosthesis rejection are (i) device weight, (ii) unsatisfactory actuation speed and (iii) lack of durability [2]. The three most important features which encourage prosthesis use are (i) the prosthesis' cosmetic appearance, (ii) ease of use, and (iii) engineering value, such as the ability to be fitted without cumbersome straps and device novelty [2]. Over $40 \%$ of the total functionality of the human hand is attributed to the thumb [3]. Therefore, an improvement in the function and realism of thumb motions in a prosthetic hand will have a major effect on the functionality of the prosthesis itself.

In spite of the important contribution of the thumb in hand motions and gestures, no significant work has been undertaken on examining the Range of Motion $(R o M)$ which a prosthetic thumb should exhibit. Whilst past research has examined the moment arms and normal forces at each thumb joint [4], [5], no work has been found which specifically quantifies the RoM of the human thumb. As such, the RoM of an actuated thumb on many myoelectric prostheses

K. Dermitzakis is with the AILab, UZH, Switzerland dermitzalifi.uzh.ch. A. Ioannides is with the BIRLab, ETHZ, Switzerland iandreas@ethz.ch. Hwai-ting Lin is with the Department of Sports Medicine, KMU, Taiwan whiting@kmu.edu.tw. is arbitrarily selected, with the thumb itself also similarly placed and shaped.

We question this design practice as we expect a significant RoM and workspace volume reduction for performing different grasps over the thumb's maximum RoMs. In order to obtain a quantifiable measure of grasps, a number of specific thumb motions are used to generate a workspace point cloud that is then compared to the point cloud generated by the maximum RoM of the thumb, as found in literature. We hypothesise that it is possible to reduce the actuated Degrees of Freedom (DOFs) of a prosthetic limb - in this case, the thumb - but maintain the RoM that will allow it to perform these grasps successfully.

\section{Human thumb MODELLING}

\section{A. Modelling Tools}

a) Denavit-Hartenberg Convention: All modelling work described herein was conducted using the MATLAB Robotics Toolbox [6]. The thumb models were created as serial kinematically-rigid robots, using the standard DenavitHartenberg $(\mathrm{DH})$ notation [7]. The coordinate transformation between two consecutive links is defined in a kinematic chain by a $4 \times 4$ homogenous transformation matrix, uniquely defined by four independent parameters, two distances and two non-planar angles. The 'standard' DH convention defines the axes at joint $(n)$ in reference to the previous joint $(n-1)$. In the interest of brevity, the details of the DH will not be presented; interested readers are directed to [7].

b) $\alpha$-Shapes: The simplest measure providing a quantitative comparison of different point clouds is their bounding volume. This can be computed using alpha-shapes, which formalise the abstract notion of a shape for a set of points. For a finite set of points $S$ in $\Re^{3}$, and a real constant $\alpha \geq 0$, a set of open-spheres of radius $\alpha$ is produced and fitted to $S$ so that every sphere is uniquely defined by two points in $S$ placed on its boundary or mathematically: $\lim _{\alpha \rightarrow 0} S_{\alpha}=S$ and $\lim _{\alpha \rightarrow \infty} S_{\alpha}=$ conv $S$. A rigorous definition of alpha shapes can $\alpha \rightarrow \infty$
be found in [8].

\section{B. Kinematic Models of the Human Thumb}

The thumb is now almost universally regarded as a threejoint, five-DoF system [4], [9]-[12], with a flexion-extension $(F E)$ and an abduction-adduction ( $A A)$ axis on each of the carpometacarpal $(C M C)$ and metacarpophalangeal $(M C P)$ joints, and a single flexion-extension axis on the interphalangeal (IP) joint. Moreover, a number of studies argue that the thumb axes are non-intesecting and non-orthogonal [11], [13], [14]. Some research articles consider the CMC and 
MCP joints as orthogonal, intersecting axes [12], [15], idealising them to universal joints. Such models have been shown to predict inaccurate thumb tip forces [16], or the abductionadduction motion of the IP joint [9].

Further, natural variation within the human population ensures that no single thumb model can be constructed. Existing Monte-Carlo simulations have shown that the anatomic variation of the position and orientation of the thumb FE and AA axes converges to a multi-modal distribution of four distinct models [11]. $65.2 \%$ of the Monte-Carlo simulations converge to a model with an MCP FE axis distal to the MCP AA axis. The other distinguishing difference lies in the common normals of the $z_{4}$ and $z_{5}$ axes.
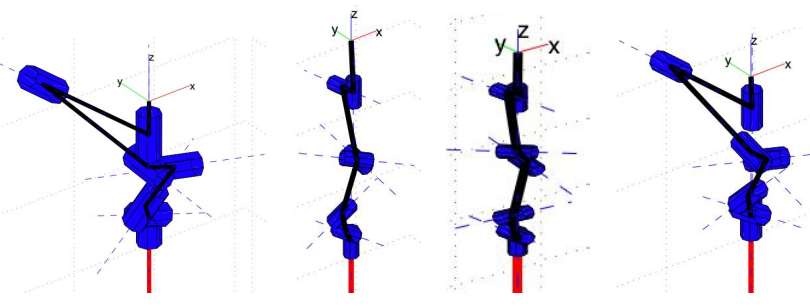

Fig. 1. The four thumb models as serial kinematic links. From left to right: Models I, II, III and IV.

The four sets of Denavit-Hartenberg parameters obtained from Santos et al. [11] were processed in MATLAB, and produced the robotic thumbs shown in Fig. 1. All models were developed with a common reference frame as 8-axis, 7 -segment serial robots. The additional segments are virtual and required to properly align the thumb axes under the DH-convention. The $z$-axis is axial to the thumb in its neutral position ${ }^{1}$. The $x$-axis is the axis of motion when the thumb joints are actuated in flexion or extension, with flexion causing a positive change in $x$, and the $y$-axis is the axis of motion under abduction or adduction, with an abduction of the thumb causing a positive change in $y$.

\section{Maximum Range of Motion}

Many studies provide a definition of the axes of the thumb joints, but fail to report a RoM about them [11], [13], [14]. A number of different studies provide a measure for the RoM of the thumb joints, but define the angular RoM either without providing an explicit definition for the orientation of the axes [5], or by assuming that the thumb joints are either 2-DoF or 3-DoF joints with orthogonal and intersecting axes [12], [17], [18].

The ranges of motion reported by Smutz et al. [5] lie within $\mu \pm \sqrt{\sigma}$, as the ones proposed by Cooney et al. [12], except for the MCP adduction extremum, which is bracketed by $107 \%(\mu+\sqrt{\sigma})$. They are also in agreement with the data presented in [17]. Comparative RoM figures have also been quoted by Li et al. [18], except for the RoM of the CMC FE axis, which is much greater at $63^{\circ} \pm 9^{\circ}$. This figure is out of range of all the above-quoted studies, and is not considered further. Thus, to represent the maximum workspace volume

\footnotetext{
${ }^{1}$ The neutral position is defined as having the thumb straight, opposed to the medial side of the hand, and parallel to the other digits of the hand.
}

of the human thumb for all four models, the mean ROM for each joint axis of the above studies will be used (Table II-D).

\section{Grasp-Based Range of Motion}

Data was obtained directly from Lin et al. [17] for the RoM needed to perform a number of grasps relating to activities in one's daily life. Six grasps were considered: (1) tip pinch, (2) palmar pinch, (3) lateral pinch, (4) cylindrical grip, (5) power grip, and (6) spherical grip. The timedependent angular data was sampled at $3 \mathrm{~Hz}$ in the original study; in order to provide a denser dataset, the original data was interpolated twice by fit of a piecewise cubic spline to produce a $9 \mathrm{~Hz}$ dataset. A piecewise cubic spline fit ensured global $C^{2}$ continuity, eliminating infinite accelerations at the joints, and producing a bio-mechanically feasible trace. The first zero-valued sampling point in each time trace was added to assess the neutral position of the thumb and was not interpolated - hence, a single $\mathrm{C} 1$ discontinuity can be observed in the interpolated time plots. The maximum and minimum angles of each DOF required to perfom the six grasping motions are tabulated in Table II-D [17].

\begin{tabular}{|c||c|c|c|c|}
\hline \multirow{2}{*}{ Joint } & \multicolumn{2}{|c|}{ Maximum RoM } & \multicolumn{2}{c|}{ Grasp RoM } \\
\cline { 2 - 5 } & Flex-Ext & Abd-Add & Flex-Ext & Abd-Add \\
\hline IP & $60^{\circ}$ to $-20^{\circ}$ & - & $49^{\circ}$ to $0^{\circ}$ & - \\
\hline MCP & $60^{\circ}$ to $-10^{\circ}$ & $15^{\circ}$ to $-15^{\circ}$ & $24^{\circ}$ to $-23^{\circ}$ & $23^{\circ}$ to $-6^{\circ}$ \\
\hline CMC & $20^{\circ}$ to $-25^{\circ}$ & $20^{\circ}$ to $-20^{\circ}$ & $16^{\circ}$ to $-8^{\circ}$ & $10^{\circ}$ to $-15^{\circ}$ \\
\hline
\end{tabular}

\section{TABLE I}

MAXIMUM AND GRASP-SPECIFIC ROMS FOR ALL THUMB JOINTS.

With the exception of the maximum MCP extension and abduction, all reported values for the grasp-based RoM are bracketed by the maximum RoM. In comparison to the maximum RoM of each joint, the grasp angular RoM required is, at worst, 3\% (MCP-AA), and, at best, $45 \%$ smaller (CMC-FE). Examining the angular RoM required to perform the grasps suggests that, in some cases, $\mu=\sqrt{\sigma}$ [17]. The variation is thus even greater than what was observed in studies documenting the maximum angular RoM, where $\sqrt{\sigma}=0.5 \times \mu$. To the authors, this suggests that different individuals may orient their hand in different configurations for a given activity, and thereby actuate their joints through different ranges, as a result of physiological variations in digit length, palm size and so on.

\section{RESUlts}

\section{A. Maximum Range of Motion}

The thumb models were actuated through their full RoM, in order to produce a point cloud containing all possible end-effector positions, as shown in Fig. 3. A sensitivity study has shown that the alpha shape volume generated with a sampling of 12 points converged to within $3 \%$. This figure is acceptable, especially when one considers the large uncertainty characterising the measurement of the angular RoM of the axes. The probe radius was then selected as $\alpha=0.5$, to produce a smooth alpha shape, assessed by visual inspection. The alpha-shapes generated for the four point clouds are shown in Fig. 2. A much-larger probe value would sacrifice the details in the surface topology in the pursue of 
smoothness, whilst a smaller probe radius would produce ragged surfaces due the discretised sampling procedure used in generating the underlying point-cloud.
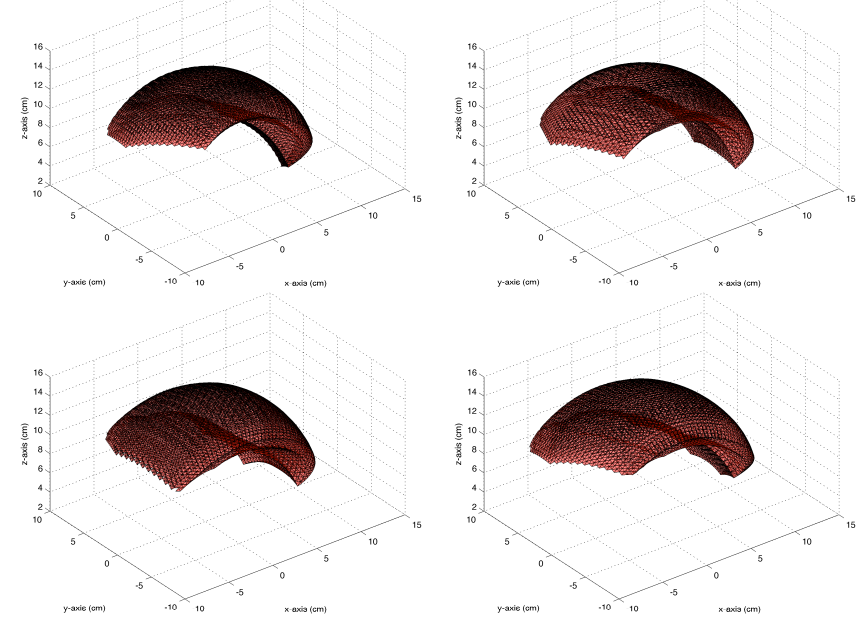

Fig. 2. Alpha-shapes for all thumb models - Maximum RoM.

\section{B. Grasp-Based Range of Motion}

Point clouds were generated using the grasp-based RoM dataset. Fig. 3 superimposes the grasp over the maximum RoM point clouds to provide a visual comparison of the extent of reduction of the optimisation target. Two views are provided: a view in the $x y$-plane, perpendicular and proximal to the axis of the thumb when in its neutral position, and a 3D view. Alpha-shapes were fitted around these sets of point clouds to quantify the workspace volume swept by the endeffector of the thumb models when performing the selected grasps. Table II compares the volumes of the bounding alphashapes for all four models, in both maximum and grasp RoMs.

\begin{tabular}{|c||c|c|c|c|}
\hline Model ID & Type I & Type II & Type III & Type IV \\
\hline \hline Volume $_{M A X}\left(\mathrm{~cm}^{3}\right)$ & 320 & 391 & 517 & 493 \\
\hline Volume $_{G R A S P}\left(\mathrm{~cm}^{3}\right)$ & 103 & 106 & 123 & 122 \\
\hline Percentage Reduction & $68 \%$ & $73 \%$ & $76 \%$ & $75 \%$ \\
\hline
\end{tabular}

TABLE II

ALPHA SHAPE VOLUMES FOR THE MAXIMUM AND GRASP-BASED ROMS.

\section{Discussion}

The four models of the human thumb produce similar point clouds, which indicate that the functional variation in the RoM in the thumbs of the human population is limited. This finding is not surprising: individuals with healthy thumbs can almost universaly perform the most common tasks requiring hand digit coordination, and therefore one expects that people with dissimilar anatomical thumb structures would share a large proportion of their RoM. However, the extrema of thumb movement are dissimilar between different thumb models. This suggests that, as is often observed, not every individual may have the ability to perform a number of strenuous grips, as a result of thumb overall length or axis orientation.
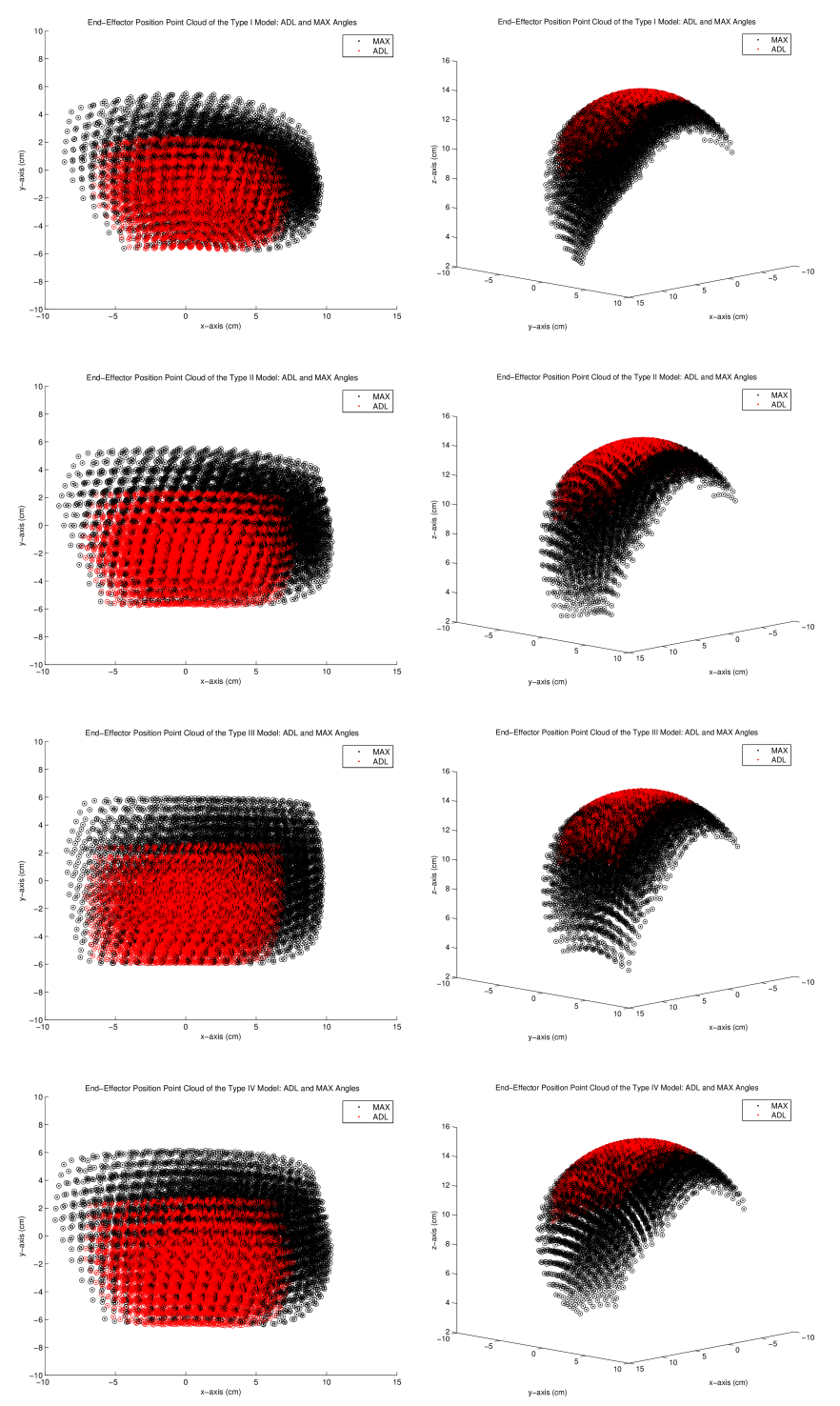

Fig. 3. Point clouds for the maximum and grasp-based RoM: Axial $(l)$ and $3 \mathrm{D}$ view $(r)$.

The natural variation in the human population prohibits the development of a single representative model for the human thumb. An actuation of the above models about a common RoM of the axes yields RoMs which are similar in shape. An attempt to quantify these yields three-dimensional polytopic shapes which appear similar in shape, but whose workspace volume can vary by up to $60 \%$. No correlation exists between the overall length of the thumb and the magnitude of its RoM; rather, it appears that the orientation of the joint axes is the defining parameter. The overlap between the RoM of different thumbs is limited. The modelling work conducted herein suggests that an individual's thumb may only be able to cover $45 \%$ of the combined RoM of a general thumb, leading to the hypothesis that a large part of the maximum RoM workspace volume of the human thumb is not used during grasp execution.

The generated alpha shapes feature a convex upper surface, 
and a concave lower surface. This can be visualised simply by sweeping one's thumb. Moreover, the alpha shapes produced only featured an outside boundary. No internal surfaces were generated, meaning that the human thumb end-effector is able to access each point in its domain within a resolution of $10 \mathrm{~mm}$. Although the joints of the thumb models were actuated through the same angles, the different anatomic structure of the four thumb caused the swept volumes of the end-effectors to vary.

\section{A. Robotic Thumb Design Implications}

The results further suggest that up to $76 \%$ of the available RoM of the human thumb is not utilised when performing simple activities, such as the six grasps considered here. This fact introduces the possibility to massively reduce the workspace volume that a prosthetic thumb needs to satisfy, bringing a number of advantages. In robotic prostheses, the reduced angular RoM allows for the use of smaller output drives (be it pulleys or gears), in turn increasing the available torque supplied by the motors while maintaining device weight. The second advantage is the possibility of optimising a robotic thumb by reducing its actuated DOFs while still satisfying the grasp-specific thumb end effector work space; this would reduce the number of actuators required, further reducing the weight and bulk of a device, whilst simultaneously increasing its autonomy and reliability.

However, the task of selecting a suitable optimisation target for the thumb is not straight-forward. All four models presented represent a certain proportion of the sample population. A union of all point clouds yields a workspace volume of $731 \mathrm{~cm}^{3}$, which is $41 \%$ greater than the Type III model volume, the largest in the selection. This is uneconomical, with such a union having little anatomic meaning. Simply combining the RoM of all four models would optimise a robotic thumb to be capable of mimicking every human thumb, which is unnecessary.

Instead, satisfying the range of motion of one human thumb would be a necessary and sufficient condition. This suggests that any one of the four models representing the human thumb can be chosen. Type II can be immediately dismissed, as it only represents $2.2 \%$ of the sample population. Of the remaining three models, Type III is the best candidate for the optimisation target, as it generates the largest workspace volume, and represents nearly one third of the sample population.

\section{B. Suggestions for Further Work}

There are still great disparities in academic research into the kinematics of the human thumb. Whilst it has been demonstrated that the revolute axes representing the human thumb joints are non-orthogonal and non-intersecting, much of the current work in documenting the RoM of the thumb joints assumes ideal orthogonal intersecting axes. Moreover, studies concerning the thumb axes orientation and RoM are carried out seperately, complicating the attempt to readacross datasets. This introduces a great deal of uncertainty into the process of modelling the human thumb. We strongly suggest that subsequent studies are carried such that the RoM of the thumb joints are documented about anatomicallyaccurate axes.

\section{ACKNOWLEDGMENTS}

The authors would like to thank Prof. Dr. Rolf Pfeifer from the AI Lab, UZH, and Prof. Dr. Fumiya Iida from the BIRLab, ETHZ for their valuable input. This research was supported by the SNSF through the National Centre of Competence in Research Robotics (NCCR Robotics).

\section{REFERENCES}

[1] K. Dermitzakis, M. R. Morales, and A. Schweizer, "Modeling the frictional interaction in the tendon-pulley system of the human finger for use in robotics." Artif Life, Nov 2012.

[2] H. Silcox III, M. D. Rooks, R. R. Vogel, and L. L. Fleming, "Myoelectric prostheses: A long-term follow-up and a study of the use of alternative prostheses," Journal of Bone and Joint Surgery, vol. 75-A, no. 12, pp. 1781-1789, December 1993.

[3] P. N. Soucacos, "Indications and selection for digital amputation and replantation," Journal of Hand Surgery, vol. 26, pp. 572-581, 2001.

[4] L. Y. Chang and Y. Matsuoka, "A kinematic thumb model for the act hand," in Proceedings of the 2006 IEEE International Conference on Robotics and Automation, Ontario, Florida, May 2006.

[5] W. P. Smutz, A. Kongsayreepong, R. E. Hughes, G. Niebur, W. P. Cooney III, and K.-N. An, "Mechanical advantage of thumb muscles," Journal of Biomechanics, vol. 31, pp. 565-570, 1998.

[6] P. I. Corke, Robotics, Vision \& Control: Fundamental Algorithms in MATLAB. Springer, 2011.

[7] R. S. Hartenberg and J. Denavit. (1964) Kinematic synthesis of linkages.

[8] H. Edelsbrunner and E. P. Mücke, "Three-dimensional alpha shapes," ACM Transactions on Graphics, vol. 13, no. 1, pp. 43-72, January 1994.

[9] D. J. Giurintano, A. Hollister, W. L. Buford, D. E. Thompson, and L. M. Myers, "A virtual five-link model of the thumb," Medical Engineering and Physics Journal, vol. 17, no. 4, pp. 297-303, 1995.

[10] M. Chalon, M. Grebestein, Wimbl ock, and G. Hirzinger, "The thumb: Guidelines for a robotic design," in The 2010 IEEE/RSJ International Conference on Intelligent Robots and Systems, Taipei, October 2010.

[11] V. J. Santos and F. J. Valero-Cuevas, "Reported anatomical variability naturally leads to multimodal distributions of denavit-hartenberg parameters for the human thumb," IEEE Transactions on Biomedical Engineering, vol. 53, no. 2, pp. 155-163, February 2006.

[12] W. P. Cooney III, M. J. Lucca, E. Y. S. Chao, and R. L. Linscheid, "The kinesiology of the thumb trapezometacarpal joint," Journal of Bone and Joint Surgery, vol. 63-A, pp. 1371-1381, December 1981.

[13] A. Hollister, W. L. Buford, L. M. Myers, D. J. Giurintano, and A. Novick, "The axes of rotation of the thumb carpometacarpal joint," Journal of Orthopaedic Research, vol. 10, pp. 454-460, May 1992.

[14] A. Hollister, D. J. Giurintano, W. L. Buford, L. M. Myers, and A. Novick, "The axes of rotation of the thumb interphalangeal and metacarpophalangeal joints," Clinical Orthopaedics and Related Research, vol. 320, pp. 188-193, November 1995.

[15] J. A. Katarincic, "Thumb kinematics and their relevance to function," Hand Clinics Journal, vol. 17, pp. 167-174, 2001.

[16] F. J. Valero-Cuevas, M. E. Johanson, and J. A. Towles, "Toward a realistic biomechanical model of the thumb: A choice of kinematic description may be more critical than the solution method or the variability/uncertainty of musculoskeletal parameters," Journal of Biomechanics, vol. 36, pp. 1019-1030, July 2003.

[17] H.-T. Lin, L.-C. Kao, H.-Y. Liu, W.-L. Wu, and F.-C. Su, "The threedimensional analysis of three thumb joints coordination in activities of daily living," Clinical Biomechanics, vol. 26, pp. 371-376, 2011.

[18] Z.-M. Li and J. Tang, "Coordination of thumb joints during opposition," Journal of Biomechanics, vol. 40, pp. 502-510, February 2007. 